This item was submitted to Loughborough's Research Repository by the author.

Items in Figshare are protected by copyright, with all rights reserved, unless otherwise indicated.

\title{
Effects of surface-functionalized aluminum nitride on thermal, electrical, and mechanical behaviors of polyarylene ether nitrile-based composites
}

\section{PLEASE CITE THE PUBLISHED VERSION}

http://dx.doi.org/10.1002/pc.23500

\section{PUBLISHER}

Wiley / @ Society of Plastics Engineers

\section{VERSION}

AM (Accepted Manuscript)

\section{PUBLISHER STATEMENT}

This work is made available according to the conditions of the Creative Commons Attribution-NonCommercialNoDerivatives 4.0 International (CC BY-NC-ND 4.0) licence. Full details of this licence are available at: https://creativecommons.org/licenses/by-nc-nd/4.0/

\section{LICENCE}

CC BY-NC-ND 4.0

\section{REPOSITORY RECORD}

Chen, Yuanming, Xuemei He, Yue Wu, Xing Gao, Jinling Wang, Wei He, Vadim Silberschmidt, and Huan Xu. 2019. "Effects of Surface-functionalized Aluminum Nitride on Thermal, Electrical, and Mechanical Behaviors of Polyarylene Ether Nitrile-based Composites". figshare. https://hdl.handle.net/2134/23613. 


\title{
Effects of Surface-Functionalized Aluminum Nitride on Thermal, Electrical, and Mechanical Behaviors of Polyarylene Ether Nitrile-Based Composites
}

\author{
Yuanming Chen, ${ }^{1,2}$ Xuemei He, ${ }^{1}$ Yue Wu, ${ }^{3}$ Xing Gao, ${ }^{2}$ Jinling Wang, ${ }^{2}$ Wei He, ${ }^{1}$ \\ Vadim V. Silberschmidt, ${ }^{2}$ Huan $\mathrm{Xu}^{4}$ \\ ${ }^{1}$ State Key Laboratory of Electronic Thin Films and Integrated Devices, University of Electronic \\ Science and Technology of China, Chengdu, China \\ ${ }^{2}$ Wolfson School of Mechanical and Manufacturing Engineering, Loughborough University, Loughborough, \\ United Kingdom
}

${ }^{3}$ Department of Mathematical Sciences, Loughborough University, Loughborough, United Kingdom

${ }^{4}$ Research and Development Department, Bomin Electronic Co., Ltd, Meizhou, China

\begin{abstract}
Aluminum nitride (AIN) with high thermal conductivity was blended in polyarylene ether nitrile (PEN) to obtain a composite system. A ball milling process could provide AIN particles of smaller size with higher surface silylation for homogeneous particle distribution in polymeric matrix. Thermal, electrical, and mechanical behaviors of the produced composites were characterized to investigate the effects of particles on the performance of PENbased composites with functionalized AIN. The composite exhibited thermal conductivity of $0.779 \mathrm{~W} \mathrm{~m}^{-1} \mathrm{~K}^{-1}$, a dielectric constant of 7.7 , dielectric loss of 0.032 , electrical resistivity of $1.39 \mathrm{G} \Omega . \mathrm{cm}$, and break strength of $36 \mathrm{~N}$ when the fraction of functionalized AIN increased to 42.3 vol\%. A fitted equation based on the improved Russell's model could effectively predict a trend for thermal conductivity of the composite systems with consideration of interfacial resistance between AIN and surrounding PEN. POLYM. COMPOS., 37:3033-3041, 2016.
\end{abstract}

\section{INTRODUCTION}

Amorphous polymers are generally modified to meet the requirements of their multifunctional applications in electronic devices. Blending process can provide an effec-

Correspondence to: W. He; e-mail: heweiz@uestc.edu.cn

Contract grant sponsor: Key Project of Science and Technology Planning of the Guangdong Province China; contract grant number: 2012A090 300007; contract grant sponsor: China Scholarship Council; contract grant number: 201306070032. tive engineering modification to enhance polymeric properties although chemical grafting reactions of polymeric chain can substantially generate new molecular crosslink structures in polymer systems. Conductive particles can be added to polymers to enhance electrical performance of polymeric composites. Silver particles with various shapes and sizes were used to form polymer-based paste for printed conductive circuits $[1,2]$. $\mathrm{BaTiO}_{3}$-filled epoxy was employed as embedded capacitor films for high dielectric constant and low capacitance tolerance [3]. In addition, strong mechanical properties could be obtained when polymer matrix was blended with carbon nanotubes [4]. Multifunctional electronic devices with high density interconnection still need polymeric substrates to rapidly transport increased heat for stable electrical performance and reliability [5]. However, amorphous polymers with phonons as main heat carriers demonstrate such low level of thermal conductivity that they are not suited for high heat dissipation at thermal design loads [6]. High conductivity of the polymer matrix is often achieved by blending highly conductive inorganic fillers [7,8].

Different inorganic ceramic fillers have been used to improve thermal conductivity of polymer matrix since such fillers could form as more as possible continuous heat conductive chains in the matrix $[6,9,10]$. Sim et al. [11] employed $\mathrm{Al}_{2} \mathrm{O}_{3}$ and $\mathrm{ZnO}$ to form conductive chains to enhance heat dissipation of silicone rubber but only a limited increase in thermal conductivity was obtained at 10 vol\% filler concentration in the composite. Zhou et al. [12] found that $\mathrm{Si}_{3} \mathrm{~N}_{4}$ particles could form continuously connected dispersion chains acting as the dominant 
thermally conductive pathways in the plastic matrix. Yang et al. [13] pointed out that thermal conductivity was increased in epoxy nanocomposites filled with nano-sized silicon carbide. Chen et al. [14] reported that $40 \mathrm{wt} \%$ of boron nitride nanoplates in boron nitride/polyimide composite was sufficient to generate large thermally conductive paths for dissipating the generated heat. Epoxy-based composites filled with hexagonal boron nitride and cubic boron nitride as hybrid filler system exhibited higher thermal conductivity than that when blending only with hexagonal boron nitride [15]. Although polyarylene ether nitrile (PEN) possesses excellent mechanical properties and thermal thermo-oxidative stability, different fillers are also added in PEN matrix for the formation of highperformance material [16-18]. In addition, PEN with low thermal conductivity needs to be modified with high thermal conductive fillers to promote its application for heat conduction. Aluminum nitride (AIN) with high thermal conductivity is a potential inorganic filler to enhance heat transport for polymeric composites [19-21]. AlN could generate thermally conductive networks for polymeric composites to cause a great increase of thermal conductivity.

In this work, functionalized AIN particles were employed to modify the performance of PEN matrix. A ball-milling process was used to further decrease the size of AlN particles and maximize the silylation at their surface for homogeneous dispersion in the polymer matrix. Thermal, electrical, and mechanical behaviors were studied to investigate the effects of functionalized AlN on PEN-based composite systems.

\section{EXPERIMENTAL}

AlN particles (unfunctionalized AlN) with hexagonal structure and a purity of $99.8 \%$ were supplied by Shandong Pengcheng Special Ceramics Ltd., China. Four grams of AlN particles were injected in a mixed solution with $90 \mathrm{ml}$ methyltriethoxysilane, $280 \mathrm{ml}$ ethanol, and $30 \mathrm{ml}$ water. Glacial acetic acid was then added into this solution to achieve a $\mathrm{pH}$ value of 3 . The mixture was ball-milled by agate balls for $24 \mathrm{~h}$ in a planetary ball mill and then centrifuged at $2000 \mathrm{rpm}$ for $15 \mathrm{~min}$. The precipitate was subsequently dried at $120^{\circ} \mathrm{C}$ for $12 \mathrm{~h}$ under vacuum to completely remove the solvent. Thereafter, asreceived AlN particles were grinded in an agate mortar and sieved to facilitate homogeneous particles. Thus, functionalized AlN was obtained.

PEN could be modified to form structural composites under melting process or solution process. At the next stage, dried PEN was dissolved in $N, N$-dimethylformamide (DMF) to achieve a $10 \mathrm{wt} \%$ content solution. The functionalized AlN particles at the concentration of 3.2, $6.8,12.3,18.3,24.2,29.9$, and 42.3 vol\% were, respectively, added into the PEN solution for $10 \mathrm{~h}$ with vigorous stirring to avoid local aggregation. The mixture solution was filtered and casted into clean glass plates at $80^{\circ} \mathrm{C}$ for $24 \mathrm{~h}$ in order to solidify the mixture after the volatility of DMF solvent. After solvent volatility, PEN/ functionalized AIN composites were obtained when cooled to room temperature. For comparison, unfunctionalized AIN particles at different concentrations were also added into PEN to form composites.

Fourier-transform infrared (FT-IR) spectroscopy (THERMO ELECTRON NICOLET 6700) was used to determine the chemical composition of AlN particles and obtained composites. Their microstructures were observed with field emission scanning electron microscope (FESEM) (HITACHI S3400). A laser granulometer (CHENGDU JINGXIN JL1197) was employed to evaluate the size distributions of functionalized AlN particles, compared with untreated ones. An X-ray diffraction (XRD) (PHILIPS X'PERT MPD) was used to monitor grain growth of PEN-based composites with different loadings of AlN particles. Gel permeation chromatography (GPC) (SHIMADZU UV2100) was employed to determine the polymeric molecular weight of tetrahydrofuran (THF)-dissolved PEN after filtering AIN precipitate from the composites dissolved in THF. A laser-flash thermal conductive measurement (NETZSCH LFA-457) was used to evaluate thermal conductivity of PEN and composites. Measurements of dielectric properties were performed for the frequency ranging from 0 to $30 \mathrm{MHz}$ on a broadband dielectric spectrometer with a highperformance frequency analyzer (AGILENT 4285A). The electrical resistivity measurements (GWINSTEK GOM$801 \mathrm{H}$ ) of PEN/AIN composites were implemented with a four-point probe technique. A peeling strength tester (ASIDA BL51A) was used to get the mechanical strength with applying force on both ends of PEN/AIN composite films. Typical specimens were cut into the dimensions of $5 \times 5 \times 0.2 \mathrm{~mm}$ for electrical and mechanical experiments.

\section{RESULTS AND DISCUSSION}

\section{Functionalization Mechanism of AlN Particles for Blending Composites}

Most inorganic fillers are immiscible in many organic polymeric materials due to poor specific interactions at organic/inorganic interphases and negligibly low combined entropy contribution to the free energy of mixing. Silane coupling agents can function at the interface of inorganic fillers to create a bridge between the reinforcement and organic polymer. Such silane functionalization that generates silane molecules at the surface of inorganic fillers can improve wettability and dispersion of inorganic fillers in the polymer matrix [6, 13, 19]. Ball milling can lead to good results with maximal dispersion of inorganic particles for their covering with silane coupling agents due to mechanical rotation and friction between inorganic particles and agate balls. During ball milling, methyltriethoxysilane successively underwent hydrolyzation, 

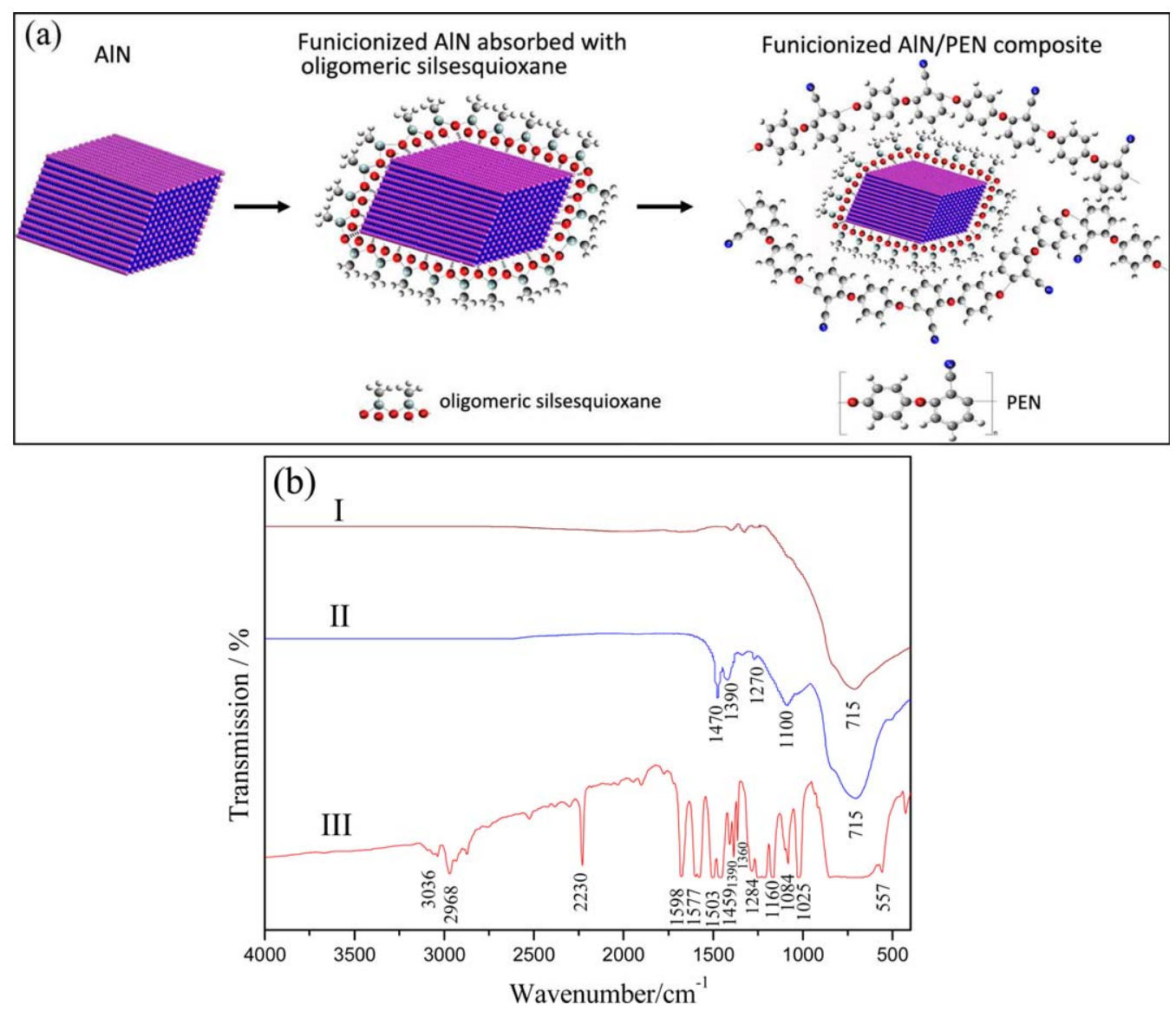

FIG. 1. (a) Schematic diagram of the preparation of functionalization of AIN particles and functionalized AlN /PEN composites; (b) FT-IR spectra of AlN (curve I), functionalized AlN (curve II), and 42.3 vol\% functionalized AlN/PEN composite (curve III). [Color figure can be viewed in the online issue, which is available at wileyonlinelibrary.com.]

dehydration, and physical absorption at the surface of AlN particles in the way of the oligomeric silsesquioxane, $\left(\mathrm{CH}_{3} \mathrm{SiO}_{1.5}\right)_{n}$, as shown in Fig. 1a. FT-IR spectra of Fig. $1 \mathrm{~b}$ allowed for the assessment of silylation of AlN particles. It was found that FT-IR spectra of functionalized AlN (curve II of Fig. 1b) presented different characteristic absorption bands, compared to that of unfunctionalized AlN (curve I of Fig. 1b). AIN exhibited a characteristic broad absorption band centered at $715 \mathrm{~cm}^{-1}$ [21]. The band at $1,390 \mathrm{~cm}^{-1}$ corresponded to the absorption peak of $-\mathrm{CH}_{3}$ while $1,470 \mathrm{~cm}^{-1}$ was assigned to the $\mathrm{C}-\mathrm{H}$ bending of $-\mathrm{CH}_{3}$ [22]. The absorption bands at 1,100 and $1,270 \mathrm{~cm}^{-1}$ corresponded to stretching vibration of siloxane $\mathrm{Si}-\mathrm{O}-\mathrm{Si}$ and flexible vibration of $\mathrm{Si}-\mathrm{C}$ [23]. Thus, AlN particles adsorbed the oligomeric silsesquioxane to generate functionalized groups for polymeric composites.

Ball milling for functionalized reaction could further reduce the mean particle size of AIN, as shown in Fig. 2. The size of functionalized AIN particles decreased to a range between 0.41 and $1.16 \mu \mathrm{m}$, compared to that between 1.06 and $3.88 \mu \mathrm{m}$ for unfunctionalized ones. $98.48 \%$ of functionalized AIN particles exhibited a mean particle size below $1.06 \mu \mathrm{m}$ after ball milling. Volume fractions of functionalized AlN particles with dominating mean particle sizes of $0.63,0.69,0.75,0.82$, and $0.89 \mu \mathrm{m}$ were $13.74,20.68,22.54,17.73$, and $10.03 \%$, respectively. This significant decrease in particle size of AlN particles resulted from mechanical grinding between the agate balls and AlN particles. Smaller particles could lead to a higher surface area and induce more unsaturated chemical bonds at the surface so that more oligomeric silsesquioxane could be absorbed at the surface of AlN particles. Thus, as shown in SEM micrographs of Fig. 3, functionalized AIN particles were dispersed better than unfunctionalized ones. Previous works indicated that a smaller particle size would result in the higher specific surface energy to induce low-level uniform particle dispersion [12, 24], thereby causing aggregation of particles. Local aggregated AIN particles (Fig. 3b) existed before their surface modification. Although particle size decreased after ball milling, functionalized AIN particles still exhibited good dispersion since their absorption with oligomeric silsesquioxane could contribute to the decrease of specific surface energy between particles to overcome van der Waals forces for aggregation [25]. In other words, formation of agglomeration could be inhibited, attributed 


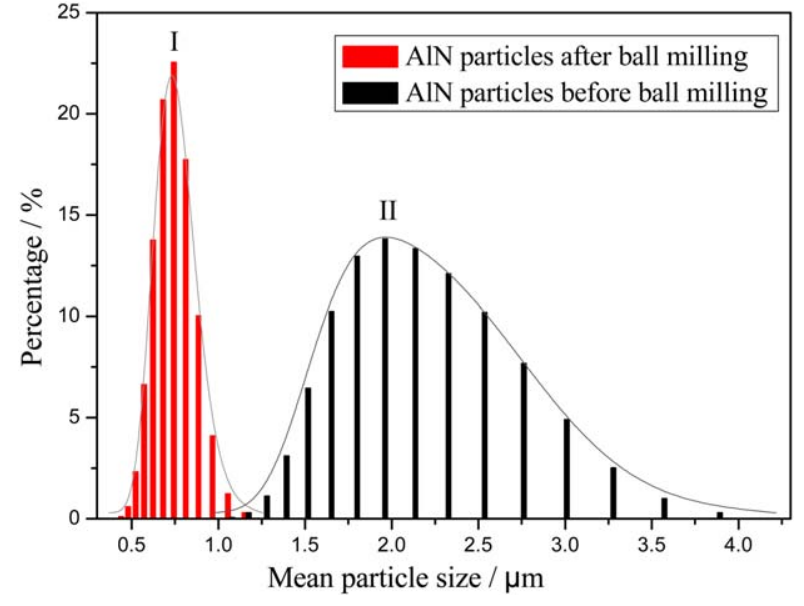

FIG. 2. Particle size distribution of functionalized AlN particles after ball milling (curve I), compared to that of unfunctionalized ones before ball milling (curve II). [Color figure can be viewed in the online issue, which is available at wileyonlinelibrary.com.]
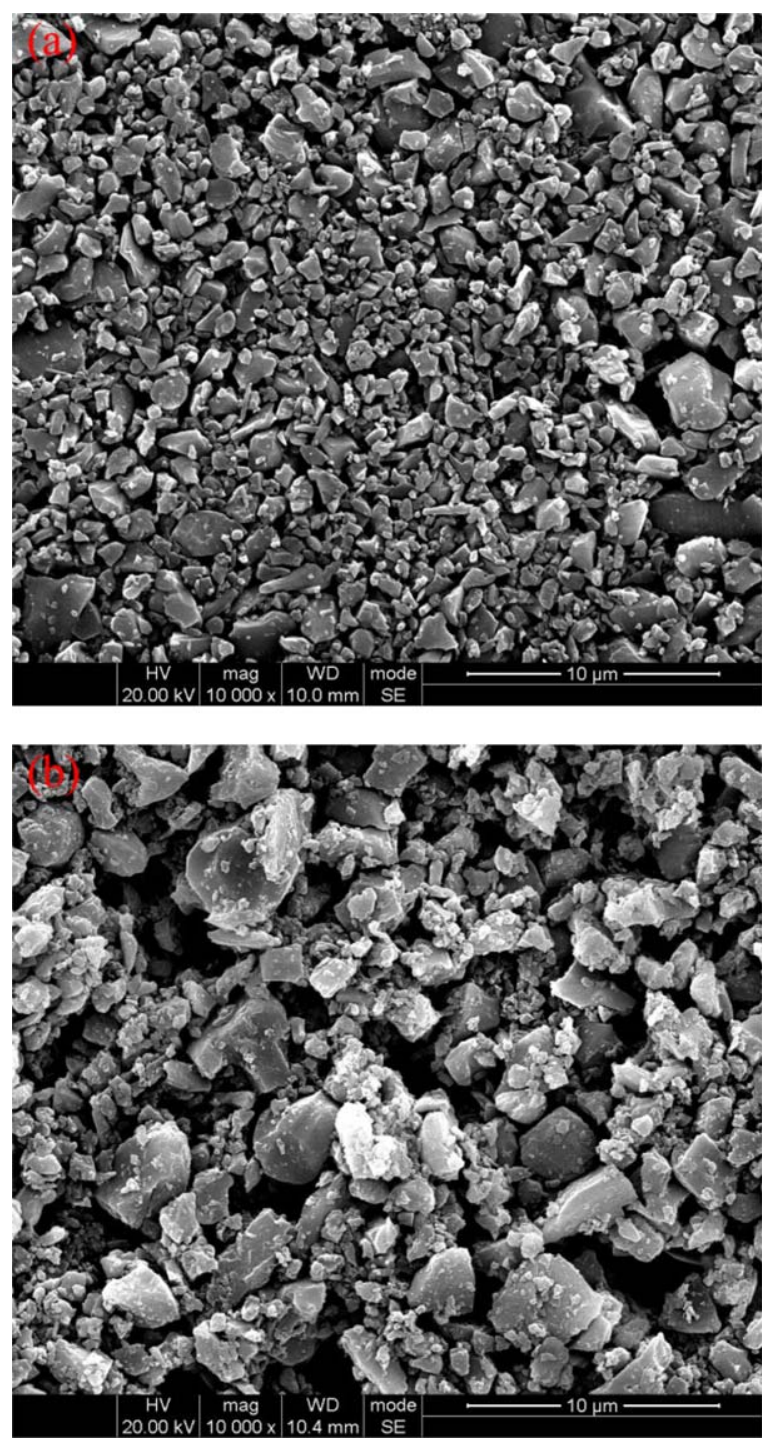

FIG. 3. SEM micrographs of (a) functionalized and (b) unfunctionalized AlN particles. [Color figure can be viewed in the online issue, which is available at wileyonlinelibrary.com.]
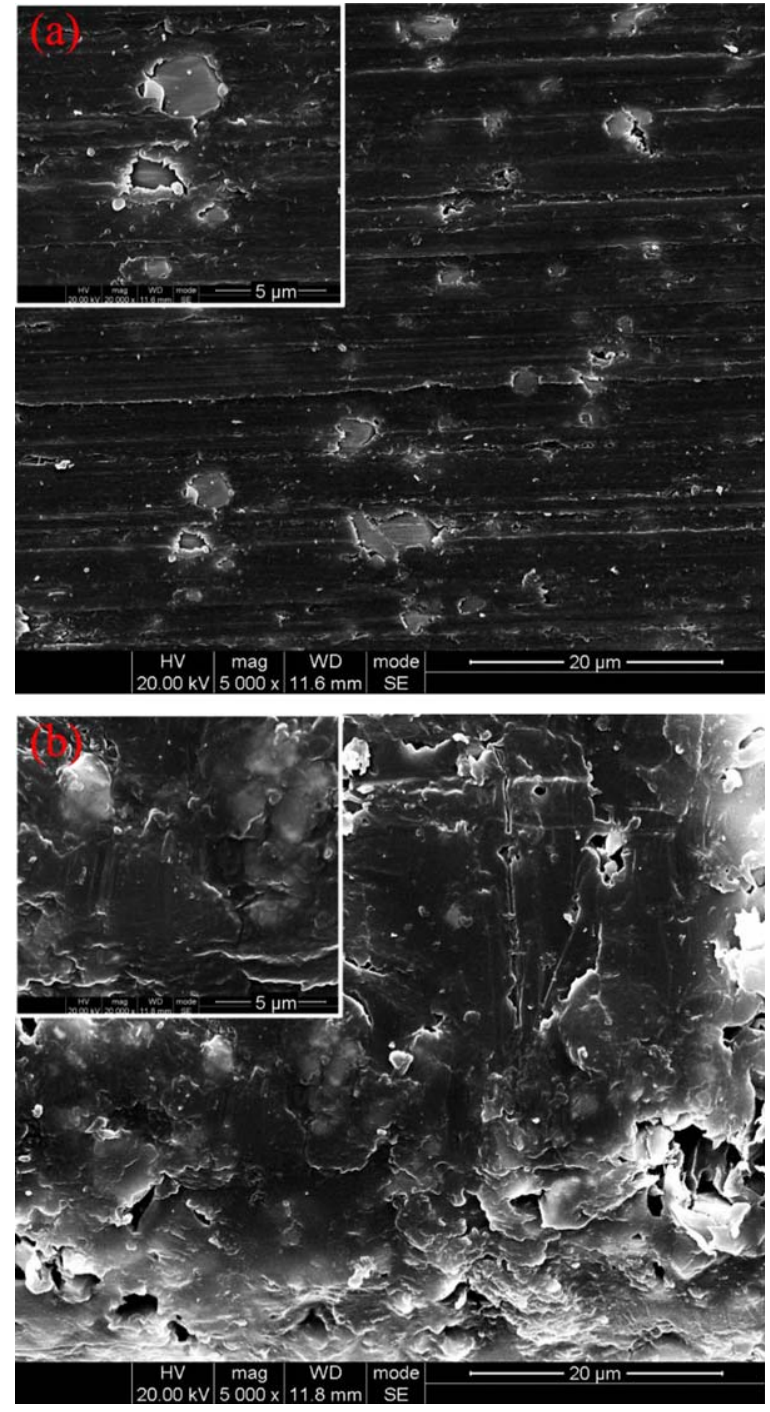

FIG. 4. SEM micrographs of PEN-based composites with (a) functionalized and (b) unfunctionalized AlN particles in 42.3 vol\% loading. [Color figure can be viewed in the online issue, which is available at wileyonlinelibrary.com.]

to the decrease of surface energy between AlN particles functionalized with oligomeric silsesquioxane.

Functionalized AIN particles were blended into PEN matrix to form composites. The measured FT-IR spectra characterized the chemical structure of functionalized AIN/PEN composite (curve III of Fig. 1b). All absorption peaks of PEN were approximately the same as those reported in literature [26]. The absorption at $557 \mathrm{~cm}^{-1}$ was attached to the phenyl characteristic absorption in PEN while the introduction of functionalized AIN particles led to a broad absorption ranging from 600 to $850 \mathrm{~cm}^{-1}$ in the way of masking the other phenyl absorption. The absorption at $1,284 \mathrm{~cm}^{-1}$ demonstrated the asymmetrical flexible vibration absorption peak of $-\mathrm{C}-\mathrm{O}-\mathrm{C}-$ while the duplex peaks at 1,160 and $1,360 \mathrm{~cm}^{-1}$ was assigned to the absorption of $\mathrm{C}-\mathrm{O}$. The absorption at 2,230 $\mathrm{cm}^{-1}$ was assigned to the symmetrical stretching of the $\mathrm{CN}$ group for PEN. The absorption 


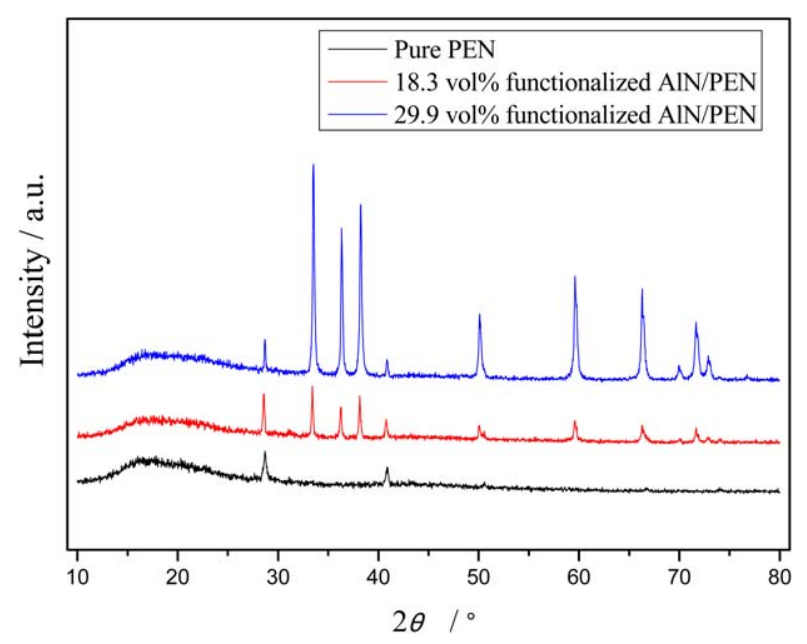

FIG. 5. XRD patterns of pure PEN and PEN-based composites with 18.3 and 29.9 vol\% functionalized AlN. [Color figure can be viewed in the online issue, which is available at wileyonlinelibrary.com.]

peaks at 1,598, 1,577, 1,503, and $1,459 \mathrm{~cm}^{-1}$ attached to benzene rings skeleton vibration peak while peaks at 3,036 and $2,968 \mathrm{~cm}^{-1}$ were the flexible vibration and the strengthened stretching vibration peak of $-\mathrm{CH}$ which linked with double bonds, respectively. The superimposed absorption ranging from 1,200 to $1,260 \mathrm{~cm}^{-1}$ was due to the presence of $\mathrm{Si}-\mathrm{C}$ in oligomeric silsesquioxane and $-\mathrm{C}-\mathrm{O}-\mathrm{C}-$ in $\mathrm{PEN}$.

Microstructure of the PEN-based composite with AIN particles was also investigated for the phase interface between AlN particles and surrounding polymeric matrix. Figure 4 presented morphological structures of the PENbased composites filled with functionalized and unfunctionalized AlN. The latter induced local aggregations separated from the continuous phase of the polymeric matrix. However, functionalized AlN particles exhibited better dispersion in the matrix, due to the presence of surficial oligomeric silsesquioxane. On the other hand, oligomeric silsesquioxane improved the interfacial adhesion when one end of the molecule ( $-\mathrm{Si}-\mathrm{O}-\mathrm{Si}-$ ) was tethered to the surface of AlN particles, and $-\mathrm{CH}_{3}$ at the other end reacted with the polymeric phase in a noncovalent way $[27,28]$. In addition, functionalized AIN particles with smaller size could also provide a larger surface area to contact with polymer, demonstrating better particle dispersion in polymer-based composite [29]. Therefore, functionalized AlN particles could be well dispersed in the PEN-based composite (Fig. 4a).

Effects of Particles on the Performance of Functionalized AlN/PEN Composites

Effect on PEN Structure. PEN with a semicrystalline structure showed diffraction peaks at $17.1^{\circ}, 28.6^{\circ}$, and $40.8^{\circ}$, as shown in Fig. 5. After adding functionalized AlN in PEN matrix, new XRD peaks appeared at $33.5^{\circ}$, $36.7^{\circ}, 38.3^{\circ}, 50.1^{\circ}, 59.7^{\circ}, 66.3^{\circ}, 70.1^{\circ}$, and $71.5^{\circ}$ in $\operatorname{good}$

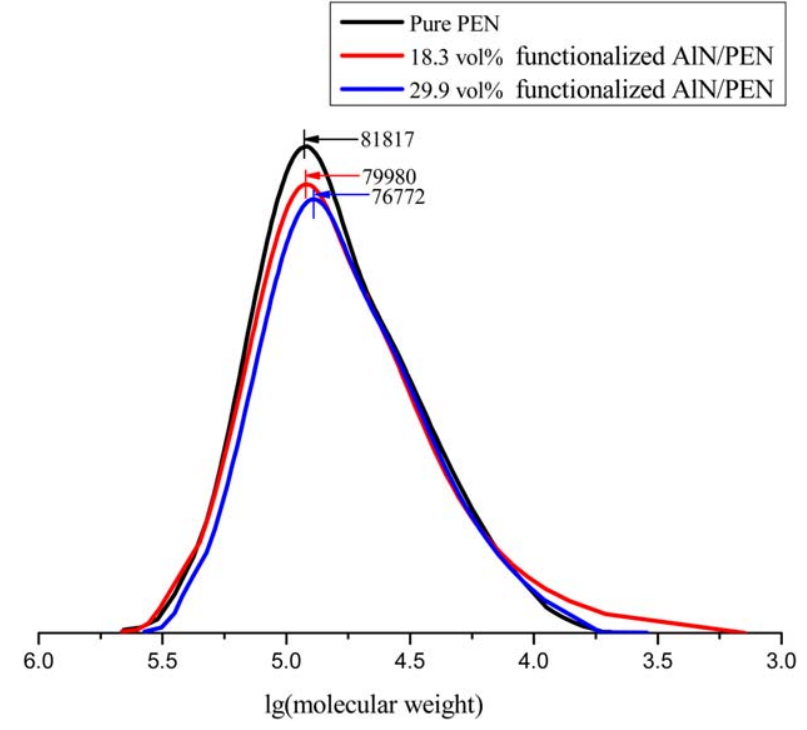

FIG. 6. GPC traces of pure PEN and PEN from composites in THF. [Color figure can be viewed in the online issue, which is available at wileyonlinelibrary.com.]

agreement with JCPDS (Joint Committee on Powder Diffraction Standards) card of AIN (JCPDS 25-1133). However, XRD patterns of functionalized AIN/PEN composites were preferentially dependent on the texture of AlN particles, and intensity of AlN peaks increased with the fraction of AIN particles in the PEN-based composites. This could be due to the existence of more AlN particles in the surface of PEN matrix for enhancing diffraction intensity when increasing AlN fraction.

As shown in Fig. 6, PEN from the composite systems with 18.3 and 29.9 vol\% functionalized AlN could retain a good cross-link structure with molecular weight ranging from 76,772 and 79,980, compared to that of pure PEN at 81,817 . This meant the polymeric molecular chains of PEN remained unchanged after DMF volatilization. Therefore, the structure of PEN molecular was stable in despite of the addition of functionalized AIN into PEN.

Effect on Thermal Conductivity. Irregular molecular chains in the polymeric system could hinder heat transport by inside phonon scattering, resulting in low thermal conductivity at $0.169 \mathrm{~W} \mathrm{~m}^{-1} \mathrm{~K}^{-1}$, as shown in Fig. 7. A significant value of percolation threshold was found when the fraction of AlN particles in PEN reached 42.3 vol\%. Thermal conductivity of the PEN-based composite with 42.3 vol\% functionalized AlN, however, increased 4.6fold compared to pure PEN, from 0.169 to $0.779 \mathrm{~W} \mathrm{~m}^{-1}$ $\mathrm{K}^{-1}$. This value was also $0.1 \mathrm{~W} \mathrm{~m}^{-1} \mathrm{~K}^{-1}$ higher than that in the composite with unfunctionalized PEN. Therefore, functionalized AIN could further improve thermal conductivity in the condition of adding more AlN particles into PEN matrix.

The decrease of particle size and the improvement in dispersion of the filler in composite systems could effectively contribute to the enhancement of thermal conductivity [30]. 
Ball milling mechanically forced the distribution of AIN particles toward the smaller sizes so that more small AlN particles could form conductive links with large ones, causing a synergistic effect for heat dissipation by phonons. For a twophase composite system, the interfacial physical contact between the polymer and filler became critical due to the sensitivity of phonons to interfacial defects [6]. Acousticimpedance mismatch induced by interfacial defects could pose an interfacial thermal barrier to heat flow, thereby inhibiting the transfer of phonon dominating heat conduction in polymer and filler and weakening the benefit of addition of highly conductive filler. However, acoustic phonons mismatch and weak bonding between the PEN matrix and unfunctionalized AIN could be addressed by introducing better developed interfacial bonding, which could be engineered by oligomeric silsesquioxane from a silylated functionalization process. On the other hand, oligomeric silsesquioxane for compact combination between polymer and particles could bridge well-dispersed AIN particles in the PEN matrix and decrease acoustic impedance to the heat flow [13]. Thus, AlN particles with silylated functionalization, small particle size, and good dispersion in the polymeric matrix could contribute to higher thermal conductivity of the PEN-based composites.

In our early work [31], many theoretical and empirical models were used to predict a trend of thermal conductivity of two-phase composite systems. However, it was difficult to identify the thermal conductivity via theoretical or empirical models due to distributed defects of filler in composite systems with thermal resistance effect. It was found that Russell's model (1) could most closely meet the trend of thermal conductivity of PEN-based composite with AlN particles by using a seriesparallel network to estimate thermal conductivity of composites:

$$
\lambda_{c}=\lambda_{p}\left[\frac{V_{f}^{2 / 3}+\frac{\lambda_{p}}{\lambda_{f}}\left(1-V_{f}^{2 / 3}\right)}{V_{f}^{2 / 3}-V_{f}+\frac{\lambda_{p}}{\lambda_{f}}\left(1+V_{f}-V_{f}^{2 / 3}\right)}\right]
$$

where $\lambda_{\mathrm{c}}, \lambda_{\mathrm{p}}$, and $\lambda_{\mathrm{f}}$ are the thermal conductivity of the composites, polymer, and filler, respectively; $V_{f}$ is the volume fraction of filler.

However, interfacial resistance between filler and surrounding polymeric matrix was not considered in the Russell's model. Accounting for the thermal resistance effect by introducing coefficient $\alpha$ to the thermal conductivity of AlN filler, the Russell's equation with undetermined coefficients was considered as (Eq. 2).

$$
\lambda_{c}=\lambda_{p} \frac{\left(a_{1} V_{f}+b_{1} V_{f}^{2 / 3}+c_{1}\right) \lambda_{f} \alpha+\left(a_{2} V_{f}+b_{2} V_{f}^{2 / 3}+c_{2}\right) \lambda_{p}}{\left(a_{3} V_{f}+b_{3} V_{f}^{2 / 3}+c_{3}\right) \lambda_{f} \alpha+\left(a_{4} V_{f}+b_{4} V_{f}^{2 / 3}+c_{4}\right) \lambda_{p}}
$$

The coefficients were obtained with Matlab Software with results as $a_{1}=0.2707, a_{2}=0.1018, a_{3}=-0.5689$, $a_{4}=0.0930, \quad b_{1}=0.2415, \quad b_{2}=0.1015, \quad b_{3}=0.4762$,

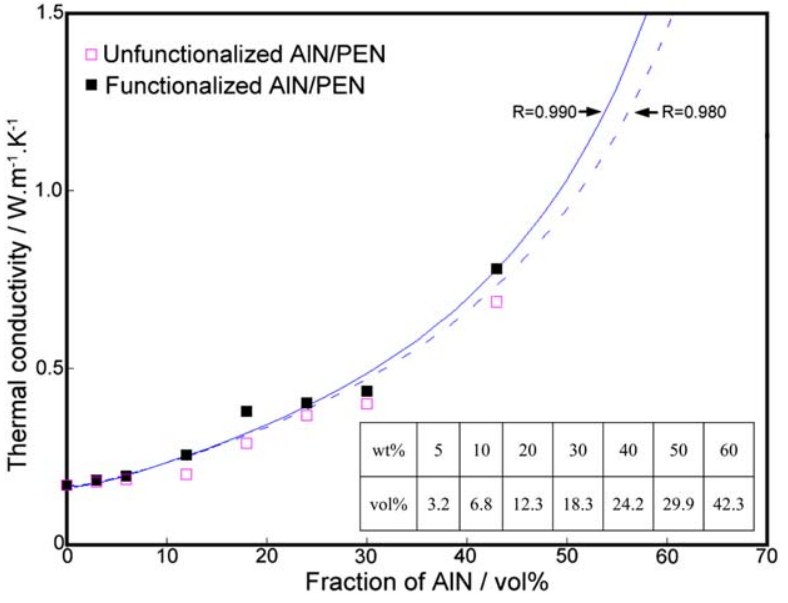

FIG. 7. Relationships between fitted equation and thermal conductivity of PEN-based composites with AlN particles. [Color figure can be viewed in the online issue, which is available at wileyonlinelibrary. com.]

$b_{4}=0.1039, c_{1}=0.0353, c_{2}=0.0993, c_{3}=0.0353$, and $c_{4}=0.0993$. The coefficients of thermal-resistance effect $\alpha$ were taken as 0.8988 for unfunctionalized AlN particles and 0.9810 for functionalized AIN particles in the composite systems, since the equivalent thermal conductivity was higher in the composites with silylated AlN. Thus, functionalized AIN exhibited a higher equivalent value of thermal conductivity, $\alpha \lambda_{\mathrm{f}}$ since silylated absorption at the surface of AlN particles reduced interfacial resistance. As shown in Fig. 7, the fitted equation with the consideration of interfacial resistance could effectively predict the trends on thermal conductivity of PEN-based composite with the addition of AlN particles in the loading of lower than $50 \mathrm{vol} \%$.

Effect on Electrical Performance. Dielectric properties of polymeric substrates in printed circuit boards play an important role in device performance with regard to electrical signals since a high dielectric constant could result in a strong negative effect on signal propagation by increasing a delay time while a high dielectric loss could induce signal attenuation to form signal distortion [12]. Figure 8 presented the obtained dielectric properties of the PEN-based composites filled with functionalized AIN at room temperature, as a function of filler fraction for different frequencies. Higher concentrations of functionalized particles resulted in higher dielectric constant of composites, as shown in Fig. 8a. At 42.3 vol\% fraction in the PEN-based composite, functionalized AIN provided a dielectric constant of about 7.7 at $0 \mathrm{~Hz}, 1.8$ times larger than that of pure PEN. AIN particles with a higher dielectric constant dominated the dielectric constant of the composites with the increased concentration. In addition, more minicapacitor networks formed between the PEN matrix and AlN particles with lower particle sizes further increased the dielectric constant of composites with higher fractions of functionalized AIN [32]. Furthermore, 

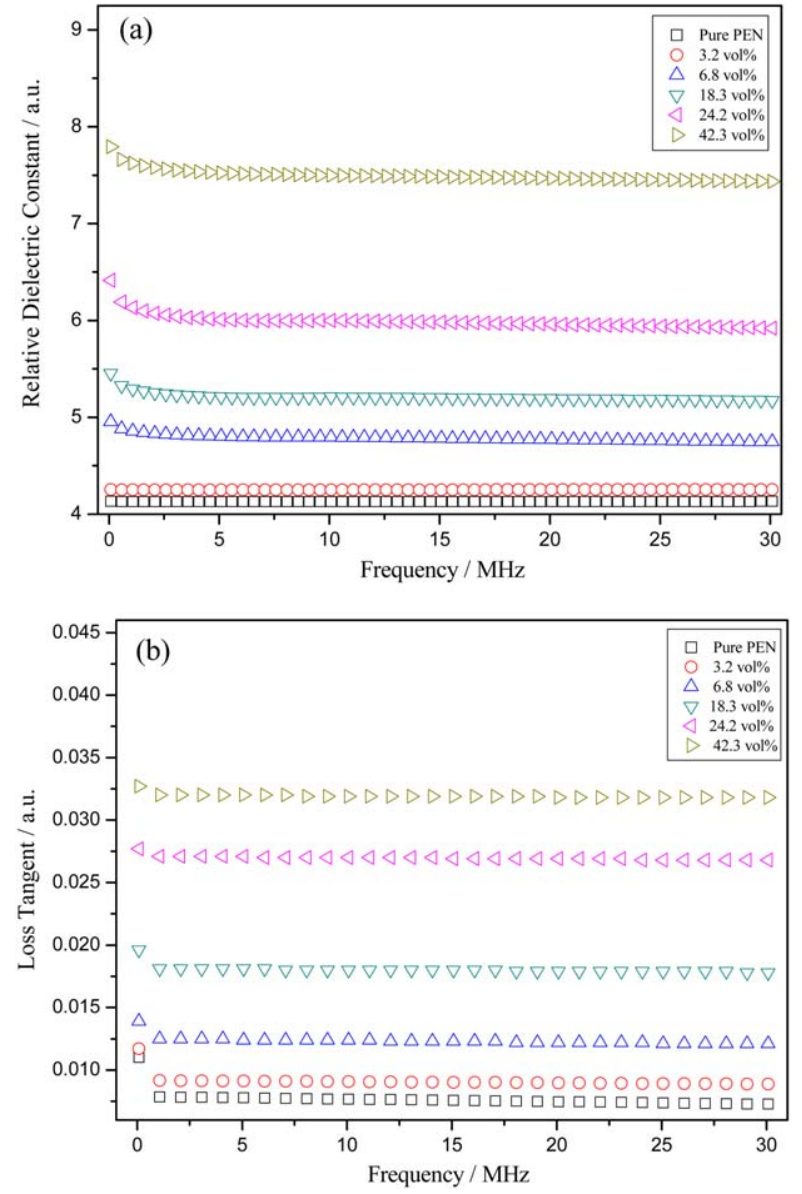

FIG. 8. Dielectric properties of pure PEN and PEN-based composites with different concentrations of functionalized AlN: (a) dielectric constant and (b) dielectric loss. [Color figure can be viewed in the online issue, which is available at wileyonlinelibrary.com.]

the filler size and microstructure of the composites (interface between the filler and matrix) could also influence the final dielectric constant of the polymeric composites with fillers [33]. With a decrease in the particle size, there was a higher chance for AlN particles to form random channels while stronger interfacial interaction between functionalized AIN and PEN matrix could be caused by silylated functionalization. A synergistic effect from the decrease in the size of particles and silylated functionalization led to a lower chance of formation of voids and pores in composites. Thus, good distribution of AlN particles in the surrounding matrix resulted in the increase of dipole-dipole interaction between the functionalized particles and the PEN matrix, thereby contributing to a higher dielectric constant [34].

However, at low frequencies, the composites exhibited significantly higher dielectric constants than at high frequencies. The dielectric constant of the composite systems reduced with the increase in frequency and was less dependent on frequency in the range from 5 to $30 \mathrm{MHz}$. This was attributed to a leading effect of electronic shift polarization that could immediately contribute to the change in the dielectric constant, rather than relaxation

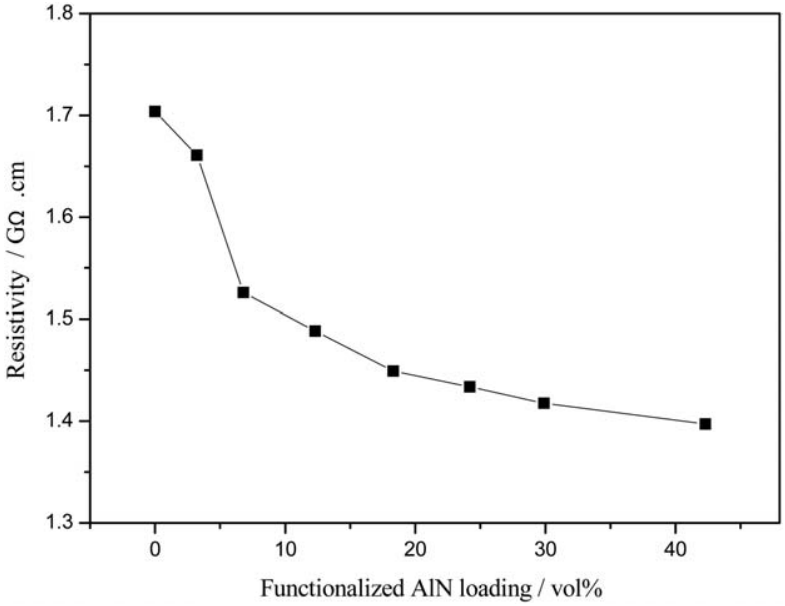

FIG. 9. Electrical resistivity plot for pure PEN and functionalized AlN/ PEN composites.

polarization that needed more time to influence this constant with the increased frequency. The earlier work [35] found that the dielectric constant of the composites at low frequencies strongly depended on dielectric properties of both the polymer and filler, while at high frequencies, it was sensitive primarily to the filler and its concentration. This indicated that at high frequency, the electronic shift polarization of polar groups in polymer chains was too slow to contribute to the dielectric constant of the composite system. Therefore, electronic shift polarization only from functionalized AlN led to the significant decrease of the dielectric constant of the composites at high frequency.

The PEN-based composites with functionalized AlN demonstrated an increasing trend of the dielectric loss with the increase of filler concentration, as shown in Fig. 8b. The dielectric loss increased to 0.032 in PEN-based composite with $42.3 \mathrm{vol} \%$ functionalized AlN, compared to 0.011 for pure PEN. This was ascribed to the dominance of AlN particles with high dielectric loss. The dielectric loss of the composites decreased at low frequencies but became almost independent on the frequency in the range from 5 to $30 \mathrm{MHz}$, similar to the trend for the dielectric constant. The relationship between the dielectric $\operatorname{loss} \tan \delta$ and the frequency $\omega$ can be defined as

$$
\tan \delta=\frac{\sigma}{\omega \varepsilon_{r}}
$$

Where $\sigma$ was the electric conductivity of free charge and $\varepsilon_{\mathrm{r}}$ is the relative dielectric constant.

The dielectric loss at a maximum value stemmed from conductance leakage at the frequency of $0 \mathrm{~Hz}$ due to absence of polarized energy consumption. From Eq. 3, the dielectric loss decreased with increasing frequency since $\omega \varepsilon_{\mathrm{r}} \gg 1$. In addition, the dielectric loss of the dielectric material resulted from relaxation polarization that would consume energy for a steady state of system 


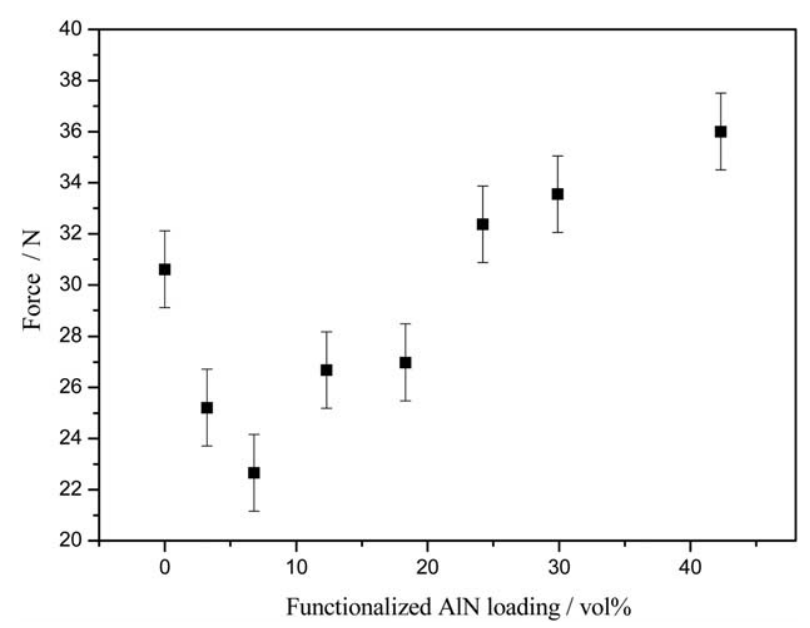

FIG. 10. Break strength for pure PEN and functionalized AlN/PEN composites.

charges. However, relaxation polarization of the polarized composite systems had less time to form the steady state at high frequency. Thus, only electronic shift polarization for no energy consumption dominated the polarized effect of the studied composite systems, thereby causing a weak dependence of the dielectric loss at high frequency.

The electrical resistivity curve for functionalized AlN/ PEN composites was illustrated in Fig. 9. Pure PEN was essentially insulative with a resistivity of $1.70 \mathrm{G} \Omega . \mathrm{cm}$ while electrical resistivity of the PEN-based composites decreased with addition of functionalized AIN. This stepwise change in resistivity was attributed to the formation of an interconnected structure of AlN particles in the composite systems. An electrical percolation threshold for electrical resistivity appeared in the composite with 6.8 vol\% functionalized AIN, resulting in a significant decrease of electrical resistivity at $1.53 \mathrm{G} \Omega \mathrm{cm}$. Electrical resistivity of the composite degraded to $1.39 \mathrm{G} \Omega \mathrm{cm}$ when 42.3 vol\% functionalized AIN were added into PEN matrix. At higher concentrations of functionalized AIN, electrons at larger percentage were permitted to flow through the composites due to the creation of easier electron tunneling by semiconductive AlN particles [36]. However, electrical resistivity of composites was less dependent on functionalized AIN at high fractions attributed to the good insulative performance of the surrounding PEN matrix.

Effect on Mechanical Performance. High loading of inorganic filler could alter the polymeric structure and hence influence the mechanical behavior of composite systems. Tensile tests were performed to obtain the break strength of pure PEN and the PEN-based composites for assessing their machinability. The break strength decreased with the increasing fraction of up to $6.8 \mathrm{vol} \%$ followed by an increase, as shown in Fig. 10. The PENbased composite with 42.3 vol\% functionalized AlN exhibited the break strength of $36 \mathrm{~N}, 6 \mathrm{~N}$ more than pure
PEN. Ruleless molecular chains in polymer could be transferred to orientation texture along tensile direction to resist the stretching force during tensile process. Addition of AlN particles at low fractions resulted in separated hampers in the composite systems to decrease local polymeric density, thereby reducing the break strength. However, AlN particles at the loading of above $6.8 \mathrm{vol} \%$ could lead to more connecting networks for load transfer between particles to inhibit the break strength. Thus, the particle interaction force and texture in the polymer provided the synergistic effect on the PEN-based composites at high fraction of AlN particles.

\section{CONCLUSIONS}

In this study, functionalized AlN particles with higher surface silylation and lower particle size distribution after ball-milling process were used to modify the performance of the PEN matrix. The surface adsorption of oligomeric silsesquioxane for functionalized AIN particles could enhance their dispersion in PEN matrix to form more conductive networks for effective heat transport. Thermal conductivity of the composites exhibited an increasing trend as the concentration of functionalized AIN was raised. Thermal conductivity of $0.779 \mathrm{~W} \mathrm{~m}^{-1} \mathrm{~K}^{-1}$ was obtained in the PEN-based composite with 42.3 vol\% functionalized AlN. The improved Russell's model with parameters obtained by cure-fitting was effective to describe the trend of thermal conductivity of the composite systems when interfacial resistance between AIN and surrounding PEN was accounted. An increase in the dielectric constant and the dielectric loss was observed in the PEN-based composites with functionalized AIN for the increase in the fraction of functionalized particles. A weak dependence on the variation of dielectric loss and dielectric loss was found in the composites when immediately increasing the frequency. The PEN-based composite with 42.3 vol\% functionalized AIN could also retain electrical resistivity of $1.39 \mathrm{G} \Omega \mathrm{cm}$ to contribute to good insulating property. More functionalized AlN could generate more interaction between particles to enhance the break strength of the PEN-based composite.

\section{REFERENCES}

1. D.Y. Shin, Y. Lee, and C.H. Kim, Thin Solid Films, 517, 6112 (2009).

2. H. Jiang, K.S. Moon, Y. Li, and C.P. Wong, Chem. Mater., 18, 2969 (2006).

3. S.D. Cho, J.Y. Lee, J.G. Hyun, and K.W. Paik, Mater. Sci. Eng. B, 1110, 233 (2004).

4. J. Sha, G. Li, X. Chen, P. Xia, R. Luo, S. Yang, T. Chen, Y. Ma, and L. Xie, Polym. Compos., DOI: 10.1002/ pc.23245, (2014).

5. Y. Chen, W. He, X. Chen, C. Wang, Z. Tao, S. Wang, G. Zhou, and M. Moshrefi-Torbati, Electrochim. Acta, 120, 293 (2014). 
6. S. Ganguli, A.K. Roy, and D.P. Anderson, Carbon, 46, 806 (2008).

7. A. Yu, P. Ramesh, X. Sun, E. Bekyarova, M.E. Itkis, and R.C. Haddon, Adv. Mater., 20, 4740 (2009).

8. Z. Han and A. Fina, Prog. Polym. Sci., 36, 914 (2011).

9. M.T. Hung, O. Choi, Y.S. Ju, and H.T. Hahn, Appl. Phys. Lett., 89, 023117 (2006).

10. T. Zhou, X. Wang, X. Liu, and D. Xiong, Carbon, 48, 1171 (2010).

11. L. Sim, S.R. Ramanan, H. Ismail, K.N. Seetharamu, and T. J. Goh, Thermochim. Acta, 430, 155 (2005).

12. W. Zhou, C. Wang, T. Ai, K. Wu, F. Zhao, and H. Gu, Compos. Part A: Appl. Sci. Manufact., 40, 830 (2009).

13. K. Yang and M. Gu, Compos. Part A: Appl. Sci. Manufact., 41, 215 (2010).

14. Y. Chen, X. Gao, J. Wang, W. He, V. V. Silberschmidt, S. Wang, Z. Tao, and H. Xu, J. Appl. Polym. Sci., 132, 41889 (2015).

15. K.C. Yung and H. Liem, J. Appl. Polym. Sci., 106, 3587 (2007).

16. L. Tong, Z. Pu, Z. Chen, X. Huang, and X. Liu, Polym. Compos., 35, 344 (2014).

17. M. Liu, K. Jia, and X. Liu, J. Appl. Polym. Sci., 132, (2015). DOI: 10.1002/app.41595.

18. Z. Chen, Y. Lei, H. Tang, J. Wei, and X. Liu, Polym. Compos., 34, 2160 (2013).

19. C.C. Teng, C.C.M. Ma, C.H. Lu, S.Y. Yang, S.H. Lee, M.C. Hsiao, M.Y. Yen, K.C. Chiou, and T.M Lee, Carbon, 49, 5107 (2011).

20. R. F. Barzic, A. I. Barzic, and G. Dumitrascu, Polym. Compos., 35, 1543 (2014).

21. J. H. Yu, J. K. Duan, W. Y. Peng, L. C. Wang, P. Peng, and P. K .Jiang, Exp. Polym. Lett., 5, 132 (2011).
22. B. Chiavarino, M. E. Crestoni, O. Dopfer, P. Maitre, and S. Fornarini, Angew. Chem. Int. Edn., 51, 4947 (2012).

23. S.W. Kuo and F.C. Chang, Prog. Polym. Sci., 36, 1649 (2011).

24. B.W. Jo, C.H. Kim, G.H. Tae, and J.B. Park, Constr. Build. Mater., 21, 1351 (2007).

25. C. Zhi, Y. Bando, C. Tang, H. Kuwahara, and D. Golberg, Adv. Mater., 21, 2889 (2009).

26. Y. Changhao, D. Hongli, G. Chunfang, L. Ping, W. Wenxuan, Z. Ming, and Q. Guanming, J. Rare Earths, 25, 20 (2007).

27. Y. Xie, C.A. Hill, Z. Xiao, H. Militz, and C. Mai, Compos. Part A: Appl. Sci. Manufact., 41, 806 (2010).

28. M. Fang, Z. Zhang, J. Li, H. Zhang, H. Lu, and Y. Yang, J. Mater. Chem., 20, 9635 (2010).

29. T. Ramanathan, A.A Abdala, S. Stankovich, D.A. Dikin, M. Herrera-Alonso, R.D. Piner, D.H. Adamson, H.C. Schniepp, X. Chen, R.S. Ruoff, S.T. Nguyen, I.A. Aksay, R.K. Prud'Homme, and L.C. Brinson, Nat. Nanotechnol., 3, 327 (2008).

30. J.W. Gu, Q. Zhang, J. Zhang, and W. Wang, Polym. Plastics Technol. Eng., 49, 1385 (2010).

31. Y. Chen, W. He, G. Zhou, Y. Hu, S. Wang, and Z. Tao, Polym. Int., 63, 546 (2014).

32. Y.J. Li, M. Xu, J.Q. Feng, and Z.M. Dang, Appl. Phys. Lett., 89, 072902 (2006).

33. M.S. Ozmusul and R.C. Picu, Polym. Compos., 23, 110 (2002).

34. Z.M. Dang, Y.F. Yu, H.P. Xu, and J. Bai, Compos. Sci. Technol., 68, 171 (2008).

35. R. Popielarz, C.K. Chiang, R. Nozaki, and J. Obrzut, Macromolecules, 34, 5910 (2001).

36. M.K. Seo and S.J. Park, Chem. Phys. Lett., 395, 44 (2004). 